\title{
Complete response to acupuncture therapy in female patients with refractory interstitial cystitis/bladder pain syndrome
}

\author{
Mehmet Giray Sönmez ${ }^{1}$, Betül Kozanhan² \\ ${ }^{1}$ Department of Urology, Meram Medical Faculty, Necmettin Erbakan University, Konya, Turkey \\ ${ }^{2}$ Department of Anesthesiology and Reanimation, Konya Education and Research Hospital, Konya, Turkey
}

\begin{abstract}
Objectives: Interstitial Cystitis/Bladder Pain syndrome (IC/BPS) is a considerable issue in urology and gynecology and unfortunately, the treatment options recommended are not fully efficient. Therefore, in this study we aimed to determine the effectiveness of acupuncture treatment in patients with refractory IC/BPS.

Material and methods: 12 refractory IC/BPS female patients received ten sessions of acupuncture twice a week. The visual analog score (VAS), interstitial cystitis symptom index (ICSI), interstitial cystitis problem index (ICPI), O'Leary-Saint symptom score (OSS), Patient Health Questionnaire (PHQ9), Pelvic pain and urgency \& frequency patient symptom scale tests (PUF) and maximum voided volume (MVV) was completed in $1^{\text {st }}, 3^{\text {rd }}, 6^{\text {th }}$ and $12^{\text {th }}$ months following the treatment.

Results: There was a statistically significant decrease in all of the scores evaluated at first month compared with the baseline. While the change in VAS score in $1,3,6$ and $12^{\text {th }}$ months were found statistically significant, measurements of ICSI, OSS and PUF scores and MVV values in the $6^{\text {th }}$ and $12^{\text {th }}$ months and ICPI and PHQ scores in the $12^{\text {th }}$ month were not found statistically significant compared to the pre-treatment period. Response to treatment for the first three months after acupuncture application was (100\%), but this ratio was measured as $33.3 \%(4 / 12)$ in the sixth month and $16.6 \%$ in the $12^{\text {th }}$ month $(2 / 12)$.

Conclusions: The results of this study suggest that acupuncture appears to be an effective, useful, non-invasive method in IC/BPS patients. It can be used as an appropriate treatment method not only in refractory but also in IC patients since it is rather advantageous compared to other treating agents.
\end{abstract}

Key words: acupuncture treatment, bladder painful syndrome, interstitial cystitis

\section{INTRODUCTION}

Interstitial cystitis (IC) or bladder painful syndrome (BPS) is defined as a clinical syndrome accompanied by chronic, recurrent suprapubic pain, increase in urination frequency and nocturia complaints increasing with bladder filling. Proven infection or significant other local pathologies are not present in this syndrome. Generally negative cognitive, behavioural, emotional and sexual syndromes and sleep disorders accompany. It is evaluated as one of the sub-types of chronic pelvic pain [1, 2]. The prevalence is estimated as $2.71 \%$ in females and $1.21 \%$ in males. It is seen 10 times more frequent in females than males. The disease occurs generally between third and fifth decades [3, 4]. Although the etiology is not completely known, autoimmune mechanisms, previous infections, stress, mast cell activation, neuropathic changes and toxic materials in urine are blamed. Disruption of the barrier and permeability in bladder mucosa due to the defects in glycosaminoglycan (GAG) layer in urothelium and exposure to toxic components of the submucosal tissue plays a role in pathogenesis [1, 3].

Many different methods are used in the treatment. Amitriptyline, antihistaminics, anticholinergics, nonsteroidal anti-inflammatory drugs, cyclosporin A, pentosan polysulphate and similar oral drugs were used but it was not 
possible to demonstrate that these drugs were effective for a long time $[1,5]$. In studies with different intravesical GAG replacement treatments (hyaluronic acid, chondroitin sulphate, hepatin) aiming the renewal of glycosaminoglycan layer, it was shown that they were successful in removing the pain by $48-71 \%$, increasing bladder capacity and recovery of sexual functions and it was observed that the symptoms were acutely relieved. But a high ratio of recurrence was reported in symptoms [3-7]. It was demonstrated that interventional applications such as bladder dilatation, Botulinum toxin A (BTX-A) application, Electromotive drug administration (EMDA), Hyperbaric oxygen, Neuromodulation application, coagulation and Transurethral resection (TUR) in patients with Hunner ulcer were useful in different levels in the treatment [1]. But in long-term follow-up, it is observed that the symptoms of the patients recur and the complaints reappear in the form of attacks. Also more than $11000 \$$ is spent yearly per patient for the medical care of IC/BPS patients in USA. Thus different treatment modalities with longer activity and less cost are required [8].

Acupuncture is an important constituent of traditional Chinese medicine which has been used for over 2500 years. Acupuncture is performed by inserting needles into specific points on the skin and its effect depends on ' $Q i$ ' which may be defined as vital energy flows in channels called meridians in the body. With acupuncture treatment, the aim is to provide the body balance by removing the energy flow blockage or interruption occurring in these channels and thereby relieving most illnesses including pain. It is stated that acupuncture application increases serotonine, beta endorphin and enkephalin levels which are endogenous opioids in the plasma and brain tissue $[9,10]$. Increase in endogen opioids in plasma and brain tissue cause analgesia, sedation, motor functions recovery and immunomodulation. It is stated that this situation has effects on providing pain control of the individual, causing him/her to feel good, be happy, have normal level of apetite and sexual drives and providing psychomotor balance [9, 10]. As it is known, interstitial cystitis is one of the subtypes of chronic pelvic pain that negative cognitive, behavioural, emotional and sexual symptoms accompany. The efficiency of acupuncture in the recovery of functional disorders such as chronic pelvic pain [11, 12], dysuria induced by benign prostate hyperplasia [13] and irritative bladder symptoms [14] were demonstrated in studies. Although use of acupuncture was accepted by urology experts in the previous decade, there is a limited number of studies investigating the efficiency of acupuncture in IC/BPS in literature [15-17]. The aim of this study was to investigate the efficiency of acupuncture in medical treatment resistant IC/BPS female patients.

\section{MATERIAL AND METHODS}

Patients who referred to Konya Training and Research Hospital Urology Clinic with symptoms lasting longer than six months such as pelvic pain, feeling of pressure and discomfort and lower urinary system symptoms (nocturia, urgency, frequency and dysuria) between 2012 and 2014 were examined and evaluated due to suspicion for IC/BPS.

IC/BPS diagnosis was excluded in patients who have pathologies such as underlying bladder cancer, urethral diverticulum, spinal cord injury, stroke, Parkinson's disease, multiple sclerosis, spina bifida, cyclophosphamide treatment, radiation treatment to pelvic area, tuberculosis affecting the bladder, uterine cancer, ovarian cancer, vaginal cancer, genital herpes, pregnancy and urinary infection. In order to clarify the diagnosis in the evaluation made for IC/BPS diagnosis, medical story (especially at least one of the symptoms lasting longer than six weeks), physical examination, urinalysis, potassium sensitivity test, hunner, presence of mucosal petechial bleeding and/or glomeration and hunner ulcer with cystoscopy, taking cystology and biopsy from bladder and cystometry/urodynamy application were used $[1,6]$. IC/BPS patients who had failed at least 24 weeks of conventional treatments (dietary changes and pelvic exercise with oral pentosanpolysulphate or tricyclic antidepressant, intravesical instillations of heparin, lidocain or hyaluronic acid, applied hydrodistantion under anestheia) defined as refractory IC/BPS.

\section{Inclusion and exclusion criteria for IC/BPS patients Inclusion criteria}

Refractory IC/BPS patients with visual analog score $\geq 4$, urinating frequency $\geq 8$ and negative urinary culture result in the last 30 days, who underwent 10 sessions of acupuncture treatment were included in the study.

\section{Exclusion criteria}

Due to the evaluations, patients who had IC/BPS diagnosis but didn't meet inclusion criteria, patients with previous acupuncture treatment, localized skin infections concerning the acupoints, use of anticoagulations and those with bleeding diathesis, completed a total of 10 acupuncture treatment sessions and whose follow-up forms were unreachable were excluded from the study.

A total of 12 volunteers who met inclusion criteria were included in the study.

\section{Intervention}

Acupuncture points were determined by an certified acupuncturists based on the Traditional Chineese Medicine (TCM) treatment methods. Acupuncture was per- 
formed with sterile, disposable steel acupuncture needles (0.25 mm $\times 25-40 \mathrm{~mm}$, Suzhou Kangnian Medical Devices Ltd. Suzhou, PRC). After the skin was cleaned appropriately with pads containing $70 \%$ alcohol, acupuncture needles were applied bilaterally (if appropriate) at SP6, SP9, ST36, LIV3, LI4, KID3, BL33 and CV4 acupoints with a depth of 1-2 cm depending on the anatomical structures below. Acupuncture application points can be seen in Figure 1, Table 1. After the sense of deqi was taken in patients, needles were left placed at these points for $20-25$ minutes. Total 10 sessions of acupuncture treatment was applied to the patients as twice a week for five weeks.

\section{Assessment}

The results of O'Leary-Saint symptom score (OSS) (20) consisting of the total of VAS: visual analog score (VAS) (19),
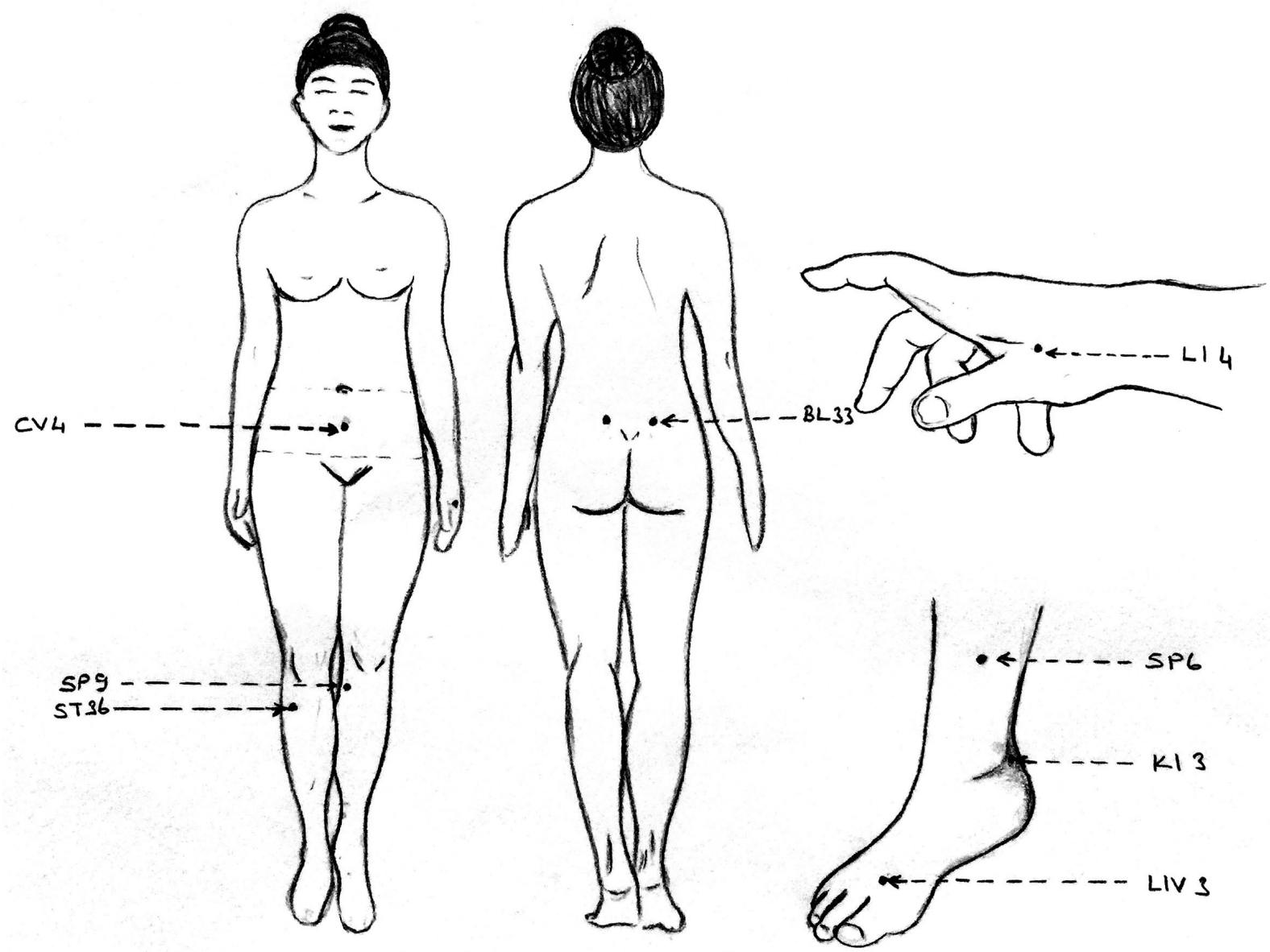

Figure 1. Acupuncture application points

\begin{tabular}{|c|c|}
\hline Acupoints & Location \\
\hline SP-6 (Sanyinjiao) & 3 tsun above medial malleolus, posterior to the medial border of tibia \\
\hline SP9 (Yinlingquan) & $\begin{array}{l}\text { On the lower border of the medial condyle of the tibia, in the depression between the posterior border of the tibia } \\
\text { and gastrocnemius muscle }\end{array}$ \\
\hline BL33 (Zhongliao) & On sacrum, in the third posterior sacral foramen \\
\hline ST36(Zusanli) & One finger width lateral from the anterior crest of the tibia, in the tibialis anteriormuscle \\
\hline LIV3 (Taichong) & On dorsum of the foot in a depression distal to the junction of the $1^{\text {st }}$ and $2^{\text {nd }}$ metatarsal bones \\
\hline LI4 (Hegu) & On the dorsum of the hand, between the $1^{\text {st }}$ and $2^{\text {nd }}$ metacarpal bones \\
\hline KI3 (Taixi) & $\begin{array}{l}\text { In depression midway between the tip of the medial malleolus and the attachment of the Achilles tendon, } \\
\text { level with the tip of the medial malleolus }\end{array}$ \\
\hline CV-4 (Guanyuan) & On the lower abdomen and on the anterior midline, 3 tsun below the centre of umbilicus \\
\hline
\end{tabular}


interstitial cystitis symptom index (ICSI), interstitial cystitis problem index (ICPI), interstitial cystitis symptom index and interstitial cystitis problem index, Patient Health Questionnaire (PHQ 9) (21), Pelvic pain and urgency \& frequency patient symptom scale tests (PUF) (22) and maximum voided volume (MVV) check made routinely when IC/BPS patients came for control and which were recorded right before acupuncture application were reached from patient files. These scores and values were recorded as pre-acupuncture treatment basal (zero time) scores and MVV value.

In the first, third, sixth and twelfth months right after the end of five-week application after acupuncture application is completed, VAS, ICSI, ICPI, OSS, PHQ9, PUF and MVV results were recorded again.

\section{Response criteria to treatment}

A decrease over 2 in VAS score, increase of $100 \mathrm{~mL}$ and above in MVV value, decrease over $30 \%$ in PUF, ICSI and ICPI scores were accepted as a response to treatment [17].

\section{Statistical analysis}

Statistical analysis was performed with SPSS, v.15.0 statistical software (SPSS, Inc., Chicago, IL, USA). Mean values of VAS, ICSI, ICPI, OSS, PHQ, PUF scores and MVV after the treatment and on follow-up at $1^{\text {st }}, 3^{\text {rd }}, 6^{\text {th }}$ and $12^{\text {th }}$, months were compared using Friedman test and Wilcoxon signed-rank test was used to evaluate the significance of differences at baseline and after treatment. $\mathrm{p}<0.05$ value was accepted as statistically significant.

\section{RESULTS}

Records of 12 female patients who underwent acupuncture treatment a total of 10 sessions for IC/BPS were analysed. Mean age of the patients was $44.1 \pm 8.2$ years (Min: 35-Max: 65), and mean duration of interstitial cystitis diagnosis time was $5.54 \pm 3.4$ years $(1-11)$ (ranging between 1 and 11 years).

There was a statistically significant decrease in all of the scores evaluated at first month compared with the pre-treat- ment. Analyses of the pre- and post-treatment scores showed a statistically significant improvement in the VAS, ICSI, ICPI, OSS, PHQ, PUF scores and MVV; $p<0.001, p<0.001$, $\mathrm{p}<0.001, \mathrm{p}<0.001, \mathrm{p}<0.001, \mathrm{p}<0.001, \mathrm{p}=0.032$ respectively. $p$ values and mean values of all parameters evaluated in the first, third, sixth and twelfth months before and after acupuncture treatment can be seen in Table 2 .

The lowest score values and highest MVV value were observed for all scores in the first month post-treatment. A gradual increase in the scores and a decrease in MVV values was observed in $3^{\text {rd }}, 6^{\text {th }}$, and $12^{\text {th }}$ months' measurements. The scores measured in the $12^{\text {th }}$ month and MVV values were rather close to the scores before treatment. While the change in VAS score in $1^{\text {st }}, 3^{\text {rd }}, 6^{\text {th }}$ and $12^{\text {th }}$ months were found statistically significant, measurements of ICSI, OSS and PUF scores and MVV values in the $6^{\text {th }}$ and $12^{\text {th }}$ months and ICPI and PHQ scores in the $12^{\text {th }}$ month were not found statistically significant compared to the pre-treatment period Pairwise comparisons of the change in $1^{\text {st }}, 3^{\text {rd }}, 6^{\text {th }}$, $12^{\text {th }}$ months compared to pre-treatment values of MVV and all scores were VAS: $p<0.001, p<0.001, p=0.002$, $p=0.01$ respectively, ICPI: $p=0.003, p=0.004, p=0.04$, $p=0.76$ respectively, ICSI: $p=0.004, p=0.009, p=0.12$, $p=0.84$ respectively, OSS: $p=0.001, p=0.002, p=0.053$, $p=0.96$ respectively, PHQ: $p=0.006, p=0.01, p=0.04$, $p=0.3$ respectively, PUF: $p=0.02, p=0.04, p=0.2, p=0.43$ respectively, MVV: $p=0.003, p=0.004 p=0.08, p=0.12$ respectively measured (Tab. 3). Response to treatment for the first three months after acupuncture application was (100\%), but this ratio was found to be $33.3 \%(4 / 12)$ in the sixth month and $16.6 \%$ in the $12^{\text {th }}$ month $(2 / 12)$.

\section{DISCUSSION}

American Urological Association defines IC/BPS as the presence of bladder related unpleasant findings (pain, pressure, discomfort) lasting over 6 weeks without the presence of infection and other definable reasons [22]. Although IC/BPS is not a life threatening disease, it was shown that quality of life measurement scores in these patients are

\begin{tabular}{|l|c|c|c|c|c|c|c|}
\hline \multicolumn{6}{|c|}{ Table 2. Mean values of all parameters evaluated before and after acupuncture treatment and $p$ values of multi analysis ( $=12)$} \\
\hline & 0 & 1 month & 3 month & $\mathbf{6}$ month & $\mathbf{1 2}$ month & p value \\
\hline VAS & $7.66 \pm 1.37$ & $2 \pm 2.6$ & $3.25 \pm 1.95$ & $5.08 \pm 1.83$ & $6.16 \pm 2.03$ & $p<0.001$ \\
\hline ICSI & $14.6 \pm 5.2$ & $8.9 \pm 4.2$ & $9.5 \pm 4.3$ & $11.5 \pm 5.2$ & $14.3 \pm 3.2$ & $p<0.001$ \\
\hline ICPI & $12.8 \pm 5.4$ & $6.25 \pm 4.49$ & $6.66 \pm 3.42$ & $9.33 \pm 3.74$ & $12 \pm 2.66$ & $p<0.001$ \\
\hline OSS & $27.5 \pm 9.4$ & $15.1 \pm 8.3$ & $16.6 \pm 7.8$ & $20.8 \pm 8.6$ & $27 \pm 5.4$ & $p<0.001$ \\
\hline PHQ 9 & $18.1 \pm 8$ & $8,3,1 \pm 8.8$ & $10 \pm 7.4$ & $12.08 \pm 7.7$ & $15.6 \pm 7.7$ & $p<0.001$ \\
\hline PUF & $22.3 \pm 8.03$ & $11 \pm 9.4$ & $14.8 \pm 7.7$ & $17.9 \pm 6.8$ & $21.6 \pm 3.9$ & $p<0.001$ \\
\hline MVV [mL] & $223 \pm 75$ & $336 \pm 88$ & $325 \pm 74$ & $266 \pm 76$ & $243 \pm 93$ & $p=0.032$ \\
\hline
\end{tabular}

*Friedman test 
Table 3. Binary statistical analysis of the change in $1,3,6,12^{\text {th }}$ months compared to the pre-treatment values of MVV and all scores ( $\mathrm{n}=12$ )

\begin{tabular}{|c|c|c|c|c|}
\hline Score & $\begin{array}{c}\text { 0-1 month } \\
\text { p value }{ }^{+}\end{array}$ & $\begin{array}{c}\text { 0-3 month } \\
\text { p value }{ }^{+}\end{array}$ & $\begin{array}{c}\text { 0-6 month } \\
\text { p value }{ }^{+}\end{array}$ & $\begin{array}{c}\text { 0-12 month } \\
\text { p value }{ }^{+}\end{array}$ \\
\hline VAS & $<0.001$ & $<0.001$ & 0.002 & 0.01 \\
\hline ICSI & 0.004 & 0.009 & 0.12 & 0.84 \\
\hline ICPI & 0.003 & 0.004 & 0.04 & 0.76 \\
\hline OSS & 0.001 & 0.002 & 0.053 & 0.96 \\
\hline PHQ 9 & 0.006 & 0.01 & 0.04 & 0.3 \\
\hline PUF & 0.02 & 0.04 & 0.2 & 0.43 \\
\hline MVV & 0.003 & 0.004 & 0.08 & 0.12 \\
\hline
\end{tabular}

+Wilcoxon signed-rank test

lower than patients with end stage renal failure who had hemodialysis treatment [23]. Acupuncture is accepted as a scientific treatment method and is commonly used in acute and chronic pain conditions. But there is a limited number of studies supporting the use of acupuncture as an effective treatment option for IC/BPS in literature [16, 17]. Honjo et al. investigated the effect of sacral acupuncture on urinary sensory dysfunction on patients whom had $O A B$ and/or suspected BPS and reported significant decrease in 24-hour frequency and VAS for pain, significant increase in maximum voided volume [24]. Katayama et al investigated the efficiency of acupuncture and moxibustion therapy on 8 patients with refractory IC and reported that there was a significant recovery in the symptoms of three patients [17].

It is considered that acupuncture is effective on IC/BPS symptoms through a few mechanisms. Afferent and efferent innervations of the bladder is formed by sympathetic nerves originating thoracic 11-lumbal 2 (T12-L2) in addition to parasympathetic and somatic nerves originating sacral 2-4 [25]. Parasympathetic system is the primary responsible system in bladder emptying. While the contraction of the bladder is provided by the parasympathetic nervous system through muscarinic receptors, sympathetic nerve system prevents the reflex activity of the detrusor and contributes to the storage of the urine during the bladder filling. The external urethral sphincter is under somatic control. The effect of acupuncture on on urination control and decreasing IC/BPS symptoms (especially urgency and pain) can be explained by inhibition in the sensory afferent nerve of the bladder, parasympathetic nerve system activation and regulation of autonomous nerve system balance [15, 25]. According to the traditional meridian theory, an acupoint has its own therapeutic property due to its specific place and related meridian. Therefore we selected the acupoints corresponding to the segmental innervation of the bladder $[26,27]$. BL33 acupoint is on the third sacral nerve root and is associated with parasympathetic nerve segmental innervation. SP6, ST36 and KI3 acupoints are located on the legs, corresponds to the innervation of skin dermatomes L4-S2 and is considered to assist bladder function by strengthening energy. While SP6 acupuncture point is preferred for urology, gynecology and genital system disorders, $\mathrm{KI} 3$ acupuncture point is the best point for urinary output.

Acupuncture is one of the standard neuromodulation therapies. Human brain is closely involved in the control of the micturation. Functional magnetic resonance imaging (MRI) studies demonstrated that there is a significant connection between brain activity and acupuncture treatment [28]. This was confirmed by Hui Wang and et al. in which acupuncture stimulation of the sacral vertebrae affects the neurons in the micturition center [29]. They showed that acupuncture suppressed bladder activity, altered the firing profiles of bladder activity-related neurons in and around Barrington's nucleus via GABAergic system mediating [29]. Similarly Hino et al. reported the result that acupuncture to the sacral periosteum improved acetic acid-induced bladder irritation in rats by inhibiting capsaicine sensitive $\mathrm{C}$-fiber activation [30]. Stimulation sacral S2-S3 vertebrae correspond to the acupoint BL33. In IC/BPS patients, we believed that brain modulation by acupuncture may also play a role in preventing the transmission of pain impulses to the brain over the spinal cord.

Ideal number of sessions and duration of treatment are not clearly established in acupuncture treatment. Thus different applications such as 6, 10 or 12 sessions are seen in studies [11, 16, 17]. Katayama et al. applied acupuncture treatment once a week for three months on 8 refractory IC patients and reported that the treatment was effective in three patients (38\%) but efficiency increased when the treatment was repeated [17]. In our study after a total of 10 sessions of acupuncture it was detected that the treatment response was quite good in the first month. In the 6th month, the change in ICSI, OSS and PUF scores and MVV values demonstrated that the efficiency of acupuncture treatment started to lose its efficiency. In the $12^{\text {th }}$ month all score values and MVV values came close to the pre-treatment values. This 
study has shown that with the application of 10 acupuncture sessions in refractory IC patients, the patients provided a complete respond for the first three months (100\%). We also think that in IC patients, repetition of acupuncture sessions after the third month will increase the efficiency of treatment and recovery time in the symptoms. Without making repetition after application, the respond rate to treatment in the $12^{\text {th }}$ month degraded to $16.6 \%$.

Our study showed that acupuncture treatment has a significant positive effect on bladder volume for the first three months. We think that this situation is parallel to the decrease in pain caused with bladder tension and the scores, increases in the score values occurring after the third month of application affected the urination volume negatively. We think that we may reach more positive results on urination volume with acupuncture treatment supported by bladder hydrotension. We also think that making prospective randomized controlled studies including a group of patients with a higher number of patients, also evaluating repetitive sessions and supported with bladder hydrotension would present the effect of acupuncture better in IC patients.

\section{CONCLUSIONS}

Acupuncture treatment is an effective, useful, non-invasive traditional method in IC/BPS patients. We think that it may be safely used even in the first diagnosis period of the disease as an appropriate treatment method not only in refractory but also in all IC patients due to not having a shown side effect and being rather advantageous due to its cost-effectiveness compared to other treating agents. But we also think that planning the treatment in repetitive sessions in appropriate patients would increase the efficiency of the treatment.

\section{ACKNOWLEDGMENTS}

This article is in accordance with ethical standards and have been approved by local authorities.The authors received no financial support for the research, authorship, and/or publication of this article.

The authors declared no potential conflicts of interest with respect to the research, authorship, and/or publication of this article. We thank to Necla Karademir who provided the acupuncture aplication points.

\section{REFERENCES}

1. Engeler D, Baranowski AP, Borovicka J. et.al. European Association of Urology Guidelines on Chronic Pelvic Pain. 2015: 23-31.

2. Bosch PC, Bosch DC. Treating interstitial cystitis/bladder pain syndrome as a chronic disease. Rev Urol. 2014; 16(2): 83-87, indexed in Pubmed: 25009448.

3. Gülpınar O, Kayış A, Süer E, et al. Clinical comparision of intravesical hyaluronic acid and hyaluronic acid-chondroitin sulphate therapy for patients with bladder pain syndrome/interstitital cystitis. Can Urol Assoc J. 2014; 8(9-10): E610-E614, doi: 10.5489/cuaj.2036, indexed in Pubmed: 25295131.
4. Hung MJ, Su TH, Lin $\mathrm{YH}$, et al. Changes in sexual function of women with refractory interstitial cystitis/bladder pain syndrome after intravesical therapy with a hyaluronic acid solution. J Sex Med. 2014; 11(9): 2256-2263, doi: 10.1111/jsm.12507, indexed in Pubmed: 24636240.

5. Lee CL, Kuo HC. Long-Term Efficacy and Safety of Repeated Intravescial OnabotulinumtoxinA Injections Plus Hydrodistention in the Treatment of Interstitial Cystitis/Bladder Pain Syndrome. Toxins (Basel). 2015; 7(10): 4283-4293, doi: 10.3390/toxins7104283, indexed in Pubmed: 26506388.

6. Kuo HC, Jiang YH, Tsai YC, et al. Intravesical botulinum toxin-A injections reduce bladder pain of interstitial cystitis/bladder pain syndrome refractory to conventional treatment - A prospective, multicenter, randomized, double-blind, placebo-controlled clinical trial. Neurourol Urodyn. 2016; 35(5): 609-614, doi: 10.1002/nau.22760, indexed in Pubmed: 25914337.

7. Engelhardt PF, Morakis N, Daha LK, et al. Long-term results of intravesical hyaluronan therapy in bladder pain syndrome/interstitial cystitis. Int Urogynecol J. 2011; 22(4): 401-405, doi: 10.1007/s00192-010-1294-y, indexed in Pubmed: 20938644

8. Robinson R. The economic burden of interstitial cystitis and painful bladder syndrome. J Urol 185(4S): e129.

9. Cabığlu MT, Ergene N. The effect mechanism of acupuncture and clinical applications. Genel Tıp Derg 2003; 13(1): 35-40.

10. Guyton AC, Hall JE. Textbook of medical physiology. Philadelphia, WB Saunders. 2001.

11. Tugcu $V$, Tas $S$, Eren $G$, et al. Effectiveness of acupuncture in patients with category IIIB chronic pelvic pain syndrome: a report of 97 patients. Pain Med. 2010; 11(4): 518-523, doi: 10.1111/j.1526-4637.2009.00794.x indexed in Pubmed: 20113410.

12. Lee $\mathrm{SH}$, Lee $\mathrm{BC}$. Electroacupuncture relieves pain in men with chronic prostatitis/chronic pelvic pain syndrome: three-arm randomized trial. Urology. 2009; 73(5): 1036-1041, doi: 10.1016/j.urology.2008.10.047, indexed in Pubmed: 19394499.

13. Li J, Han $\mathrm{CH}$, Cheng $\mathrm{XH}$, et al. [Observation on therapeutic effects of elongated needle therapy on dysuria induced by benign prostatic hyperplasia]. Zhongguo Zhen Jiu. 2008; 28(10): 707-709, indexed in Pubmed: 18972723.

14. Kelleher C, Filshie J, Burton $G$, et al. Acupuncture and the treatment of irritative bladder symptoms. Acupuncture in Medicine. 1994; 12(1): 9-12, doi: 10.1136/aim.12.1.9.

15. Tempest $\mathrm{H}$, Reynard J, Bryant $\mathrm{RJ}$, et al. Acupuncture in urological practice--a survey of urologists in England. Complement Ther Med. 2011; 19(1): 27-31, doi: 10.1016/j.ctim.2010.10.001, indexed in Pubmed: 21296264.

16. Geirsson G, Wang YH, Lindström S, et al. Traditional acupuncture and electrical stimulation of the posterior tibial nerve. A trial in chronic interstitial cystitis. Scand J Urol Nephrol. 1993; 27(1): 67-70, indexed in Pubmed: 8493470.

17. Katayama Y, Nakahara K, Shitamura T, et al. [Effectiveness of acupuncture and moxibustion therapy for the treatment of refractory interstitial cystitis]. Hinyokika Kiyo. 2013; 59(5): 265-269, indexed in Pubmed: 23719132.

18. Wewers ME, Lowe NK. A critical review of visual analogue scales in the measurement of clinical phenomena. Res Nurs Health. 1990; 13(4): 227-236, indexed in Pubmed: 2197679.

19. O'Leary MP, Sant GR, Fowler FJ, et al. The interstitial cystitis symptom index and problem index. Urology. 1997; 49(5A Suppl): 58-63, indexed in Pubmed: 9146003.

20. Löwe B, Kroenke K, Herzog W, et al. Measuring depression outcome with a brief self-report instrument: sensitivity to change of the Patient Health Questionnaire (PHQ-9). J Affect Disord. 2004; 81(1): 61-66, doi: 10.1016/S0165-0327(03)00198-8, indexed in Pubmed: 15183601.

21. Parsons C, Dell J, Stanford E, et al. Increased prevalence of interstitial cystitis: previously unrecognized urologic and gynecologic cases identified using a new symptom questionnaire and intravesical potassium sensitivity. Urology. 2002; 60(4):573-578, doi: 10.1016/s0090-4295(02)01829-0.

22. Hanno PM, Burks DA, Clemens JQ, et al. Interstitial Cystitis Guidelines Panel of the American Urological Association Education and Research, Inc. AUA guideline for the diagnosis and treatment of interstitial cystitis/bladder pain syndrome. J Urol. 2011; 185(6): 2162-2170, doi: 10.1016/j.juro.2011.03.064, indexed in Pubmed: 21497847.

23. Chancellor MB. A Multidisciplinary Consensus Meeting on IC/PBS: Outcome of the Consensus Meeting on Interstitial Cystitis/Painful Bladder Syndrome, February 10, 2007, Washington, DC. Rev Urol. 2007; 9(2): 81-83, indexed in Pubmed: 17592542.

24. Honjo H, Kamoi K, Naya $\mathrm{Y}$, et al. Effects of acupuncture for chronic pelvic pain syndrome with intrapelvic venous congestion: prelimi- 
nary results. Int J Urol. 2004; 11(8): 607-612, doi: 10.1111/j.1442-2042.2004.00868.x, indexed in Pubmed: 15285750.

25. Sung $L$, Jiaqi W. Acupuncture for urinary incontinence in adults without neurological disease. Cochrane Database of Systematic Reviews. 2006, doi: 10.1002/14651858.cd006235.

26. Liu Z, Liu B, Yang T, et.al. Clinical study of electroacupuncture treatment of senile urge urinary incontinence. Int J Clin Acupunct 2002; 13(4): 255-262.

27. Emmons SL, Otto L. Acupuncture for overactive bladder: a randomized controlled trial. Obstet Gynecol. 2005; 106(1): 138-143, doi: 10.1097/01. AOG.0000163258.57895.ec, indexed in Pubmed: 15994629.
28. Napadow V, Lee J, Kim J, et al. Brain correlates of phasic autonomic response to acupuncture stimulation: an event-related fMRI study. Hum Brain Mapp. 2013; 34(10): 2592-2606, doi: 10.1002/hbm.22091, indexed in Pubmed: 22504841.

29. Wang $\mathrm{H}$, Tanaka $\mathrm{Y}$, Kawauchi $\mathrm{A}$, et al. Acupuncture of the sacral vertebrae suppresses bladder activity and bladder activity-related neurons in the brainstem micturition center. Neurosci Res. 2012; 72(1): 43-49, doi: 10.1016/j.neures.2011.09.007, indexed in Pubmed: 22001760.

30. Hino $\mathrm{K}, \mathrm{Honjo} \mathrm{H}, \mathrm{Nakao} \mathrm{M}$, et al. The effects of sacral acupuncture on acetic acid-induced bladder irritation in conscious rats. Urology. 2010; 75(3): 730-734, doi: 10.1016/j.urology.2009.04.025, indexed in Pubmed: 19592069. 